\title{
La produzione scientifica italiana attaverso un'analisi degli articoli pubblicati sulle riviste infermieristiche: 2003-2009
}

\author{
The status of the art of scientific articles in Italy between 2003 -2009: an analysis of articles \\ published by nursing Italian journals
}

\author{
Anna Rita Marucci ${ }^{1} \quad$ Walter De Caro ${ }^{2} \quad$ Maria Rivoli $^{3} \quad$ Tommaso Trezza $^{4}$ \\ Domenico Miriello 5 Julita Sansoni 6
}

\begin{abstract}
RIASSUNTO
Il presente articolo analizza la produzione scientifica italiana attraverso una fotografia delle pubblicazioni infermieristiche su 5 riviste nazionali nel periodo tra il 2003 e 2009. Questo è il terzo articolo di un lavoro iniziato nel1978 e che ha visto due pubblicazioni nel 2005. Il lavoro ha l'intento di monitorare nel tempo la produzione bibliografica italiana per quanto attiene 5 Riviste di settore.
\end{abstract}

Gli articoli sono stati catalogati in base a criteri predefiniti con lo scopo di verificare lo status dell'avanzamento dei lavori di ricerca e di sviluppo teorico. I risultati ottenuti dimostrano che, nonostante ci sia un incremento delle pubblicazioni che applicano metodi di ricerca, queste rimangono comunque al di sotto della tendenza internazionale e che, in ambito nazionale, si prediligono ancora argomenti quali piani assistenziali, normative ed organizzazione del lavoro invece che ricerca o evidenze scientifiche.

Da un lato si tende a sensibilizzare il mondo infermieristico per essere al passo con i tempi e con il dibattito internazionale, dall'altro si registra la mancanza di adeguati strumenti per la conoscenza ed investimenti specifici nella ricerca, Il risultato è di limitare fortemente l'aggiornamento continuo scientifico nelle diverse generazioni di professionisti e la partecipazione per la generalizzazione delle stesse.

Altro risultato che può essere interpretato anche come una conseguenza, è relativo alla scarsa produzione nella letteratura italiana presa in considerazione, di Modelli Concettuali e Teorie. Modelli e Teorie che costituiscono e contribuiscono al sapere specifico di una disciplina e che si pongono quali strumenti necessari per creare un sapere e di conseguenza una immagine delle scienze infermieristiche diversa da quella di esclusiva scienza applicata.

Parole Chiave: articoli scientifici, riviste italiane, immagine infermieristica, ricerca infermieristica

ABSTRACT

This study investigates the number of articles published by Italian nursing journals by analyzing five Italian journals between 2003 and 2009. This is the third part of a study started in 1978, two articles were already published in 2005. The work is aimed at monitoring the number of nursing articles published. The articles were cataloged according to predefined criteria with the main aim to verify the status of the progress of research and theoretical development among Italian nurses. Results show that, although there is an increase in publications which apply research methods, these are still below the international trend and that, at national level, nurses prefer topics such as care plans, regulations and organization of work rather than research or scientific evidence.

On the one hand there is a raise on Italian nurses of interest in wider topic in order to be closer to the international debate, on the other hand there is a lack of adequate tools to improve knowledge and specific investments in research. As result of this there is a strong limitation in the knowledge growth of Italian nurses together with a lack of participation among and for different generations of nurses. In addition to the above it has been enucleated a scarcity within the literature analyzed of development of theoretical models, theories and concepts. Conversely theoretical models, theories and concepts are important pillars of scientific knowledge and they have a strong role in professional development, moreover these are necessary in order to set and improve nursing and nurses image nowadays far from the exclusive applied science.

Key words: scientific articles, italian journal, nursing image, nursing research

INTRODUZIONE

T a ricerca nello specifico ambito è lo strumento in base al quale si sviluppa ogni disciplina diventando

1 Coordinatore Infermieristico, UOC Pediatria ed Ematologia Pediatrica, Azienda Ospedaliera San Camillo Forlanini

Unità di Ricerca Infermieristica, Sapienza Università di Roma Corrispondenza: ar.marucci@gmail.com

2 Unità di Ricerca Infermieristica, Sapienza Università di Roma

3 Infermiere, Gruppo Operativo Comitato Infezioni Ospedaliere (CIO) Azienda Policlinico Umberto I di Roma

4 Infermiere, Ospedale Pediatrico Bambino Gesù, IRCCS, Roma

5 Studente Laurea Magistrale Scienze Inf./Ost., Sapienza Università di Roma

6 Prof. Associato Scienze Infermieristiche, Unità di Ricerca infermieristica, Sapienza Università Roma così visibile e riconosciuta attraverso la generalizzabilità e la discussione dei suoi risultati. Ogni disciplina pone quindi la ricerca quale presupposto per la propria esistenza. Partendo da questo assunto è facile dedurre che la ricerca sia una componente essenziale di tutte le discipline, quindi anche di quella infermieristica.

In tal senso non vi è alcun dubbio che il grado di affermazione di una professione e di una disciplina sia strettamente correlato all'esistenza di una letteratura che riguardi il proprio ambito specifico e che pertanto, le pubblicazioni scientifiche rappresentino il più importante strumento di trasmissione della conoscenza 
(Marucci et al, 2005). La diffusione dei risultati della ricerca attraverso Riviste di settore risulta quindi fondamentale al fine di creare una solida letteratura ed il conseguente scambio culturale oltre a far scaturire discussioni utili anche per la condivisione e la generalizzabilità.

$E^{\prime}$ in questa ottica, proseguendo i lavori pubblicati nel 2005 (Sansoni et al; Marucci et al.) che analizzano la letteratura infermieristica italiana dal 1978 al 2003 che il presente articolo vuole fornire un analisi qualiquantitativa di ciò che hanno pubblicato gli infermieri italiani dal 2003 al 2009. Lo scopo è stato quello di verificare l'andamento degli articoli rappresentati da studi di ricerca e contribuire a delineare una nuova fotografia del momento storico professionale. Inoltre vuole fornire la possibilità di confronto con i risultati degli anni presi in esame precedentemente.

In analogia, nel presente lavoro si è utilizzato un metodo descrittivo retrospettivo utilizzando le medesime categorie e parametri prestabiliti con la modifica di alcune varianti che verranno più avanti esplicitate.

\section{REVISIONE DELLA LETTERATURA}

Sull'argomento in esame sono state prodotte diverse pubblicazioni sia in Italia che a livello internazionale. Nel 2001 è stato evidenziato come ad una analisi dei lavori di tesi di primo livello di infermieristica, su 295 elaborati tra il 1976 e il 1996, il 30\% della distribuzione riguardasse l'ambito assistenziale, il 40\% quello formativo e il $26 \%$ quello organizzativo e di management (Cantarelli, 2001).

Rimanendo in ambito nazionale oltre che al progetto già citato, si sono pronunciati Zanotti e Pecile (2003) mostrando che, in un campione di circa 300 studi datati fra il 1983 e il 2001, gli infermieri italiani hanno prodotto lavori riguardanti principalmente l'assistenza attraverso modelli di indagine osservazionale su campioni di convenienza, mettendo in luce l'importanza di una formazione specifica nel campo della ricerca per poter dare validità al ruolo del nursing. Nonostante sia stato dimostrato un incremento delle ricerche pubblicate sulle riviste infermieristiche nazionali (Sansoni et al, 2006), in Italia, negli stessi anni, si pubblica meno rispetto al resto d'Europa. Ciò viene anche evidenziato in un lavoro che metteva a confronto 2 riviste Infermieristiche Europee con 2 riviste infermieristiche Italiane censite - dimostrando una prevalenza di articoli di ricerca (qualitativi e quantitativi) nettamente a favore delle riviste Europee (Sansoni et al., 2006). Questi risultati evidenziano la necessità di un più ampio ed approfondito dibattito scientifico a livello nazionale e di un indispensabile aumento di ricerche nello specifico ambito professionale al fine di avvalorare la disciplina infermieristica con conseguente miglioramento dell'assistenza.
In letteratura si parla sempre più di Evidence based nursing per indicare la sistematica interconnessione di evidenze prodotte scientificamente con la tacita conoscenza di un esperto ricercatore per ottenere un cambiamento nella pratica (French, 1999); e come autori diversi (Hunt 1996, Kitson 1996, Burrows 1995) affermino che molte pratiche infermieristiche siano fondate su esperienza, tradizione, intuizione e teorie invalidate e che la mancanza di sviluppo infermieristico nella ricerca sia causato sostanzialmente da alcuni fattori quali: 1) non conoscenza della ricerca, 2) non comprensione della stessa, 3) non convinzione dell'utilità 4) incapacità metodologica 5) impossibilità dell'utilizzo dei risultati.

Attualmente grazie alla progressiva diffusione di modelli di ricerca in ambito infermieristico e alle innovazioni strutturali in ambito formativo, si riserva sempre più attenzione all'aggiornamento culturale dei professionisti infermieri e di conseguenza, anche nella pratica clinica vengono introdotti $\mathrm{i}$ risultati ottenuti dalla ricerca. A livello europeo un contributo recente e significativo è stato dato dalla letteratura irlandese. Attraverso un'analisi dei lavori di ricerca pubblicati dagli infermieri nel periodo 1990-2005, in base ai criteri di inclusione (qualità del progetto di ricerca, campionamento, analisi dei dati, autore ) sono stati selezionati 152 articoli da un totale di 213 studi pubblicati. Le aree principali di ricerca hanno riguardato la pratica clinica $(56 \%)$, la formazione professionale degli infermieri (25\%), il 19\% management infermieristico e le questioni professionali (Mc Carthy, 2006).

Come già è stato evidenziato sopra, attraverso l'analisi della letteratura prodotta in uno specifico settore è possibile avere un'immagine dell'evoluzione culturale e dell'elaborazione scientifica della stessa. Negli ultimi dieci anni a livello internazionale sono stati condotti studi in realtà differenti che hanno dato risultati discordanti tra loro. Ad esempio, alcuni ricercatori brasiliani hanno svolto una indagine nel periodo tra il 2005 e il 2006 su due riviste infermieristiche internazionali e su un totale di 108 articoli esaminati le aree tematiche maggiormente studiate risultavano essere la formazione $(19 \%)$, la salute collettiva e quella negli adulti entrambe con l'11\% (Silva et al, 2008).

Uno studio simile è stato condotto da Polit (2009) ed ha sviluppato un' analisi di 1072 articoli pubblicati su otto importanti testate di ricerca, sempre tra il 2005 e il 2006. Questo lavoro ha evidenziato differenze significative nelle diverse regioni del mondo in riferimento alle varie categorie. In Europa, Canada e Australia risultano prevalenti gli studi relativi ai professionisti (infermieri) mentre in Asia e negli Stati Uniti è più evidente, come fulcro della ricerca, un interesse per i pazienti. Questo dato potrebbe essere interessante se messo in relazione a quanto affermato da Meleis (2011) quando dichiara che nello sviluppo di una scala della ricerca, 
all'inizio si tende a studiare sé stessi come professione, la propria preparazione e organizzazione prima di passare all'assistenza dei pazienti ed alla definizione dei propri modelli. Sempre Polit, ha messo in evidenza che Nazioni come Norvegia, Svezia e Regno Unito prediligono studi di ricerca attraverso metodi qualitativi. Troviamo interessante anche questo dato in quanto pensiamo che ove ci sia evidente necessità di sviluppo disciplinare e professionale, siano più utilizzati i metodi di ricerca qualitativi in quanto strumenti importanti per la costruzione di sapere e di modelli specifici sui quali validare le proprie conoscenze.

L'analisi condotta sugli stessi articoli ha sottolineato le differenze dell'evoluzione della ricerca Infermieristica nei Paesi messi a confronto. Pertanto si può affermare che al momento la produzione di sapere infermieristico scientifico quale specchio riflesso di diverse realtà culturali e scenari in cui si evolve la professione infermieristica nei vari Paesi del mondo, non sia omogenea.

\section{MATERIALI E METODI}

Il metodo scelto - retrospettivo - è sovrapponibile a quello utilizzato nel progetto già citato del 2005 . Le cinque riviste analizzate dal 2003 al 2006 sono Professioni Infermieristiche, Assistenza Infermieristica e Ricerca (AIR), Scenario, Nursing Oggi e l'Infermiere.

Per gli anni dal 2003 al 2006 sono stati analizzati 112 fascicoli per un totale di 887 articoli di cui 707 scritti da infermieri e 180 da Autori appartenenti ad altre professioni (media 221.7 articoli anno), suddivisi come riportato nelle la Tab. 1

Il lavoro si è articolato in diverse fasi:

o Ricerca ed acquisizione di tutti i numeri delle riviste inserite nel campione

o Lettura ed analisi di tutti gli articoli

o Codifica delle diverse tipologie di lavori per categorie e sottocategorie precedentemente stabilite (Fig.1)

o Classificazione degli articoli con particolare attenzione alla qualifica degli autori

o Classificazione degli articoli in base alla loro struttura (se rispondente al format di un articolo di ricerca)

o Elaborazione dei dati (strutturazione di un database specifico)

o Decodifica, lettura e discussione dei dati analizzati

Il database è stato creato utilizzando un foglio di calcolo Excel. Ad ogni riga corrisponde un singolo articolo che è stato classificato secondo diversi parametri, l'individuazione delle categorie e sottocategoria di appartenenza ha consentito successivamente di attribuire ad esse un codice numerico che permettesse di raggruppare gli articoli presi in esame (Tab. 3).

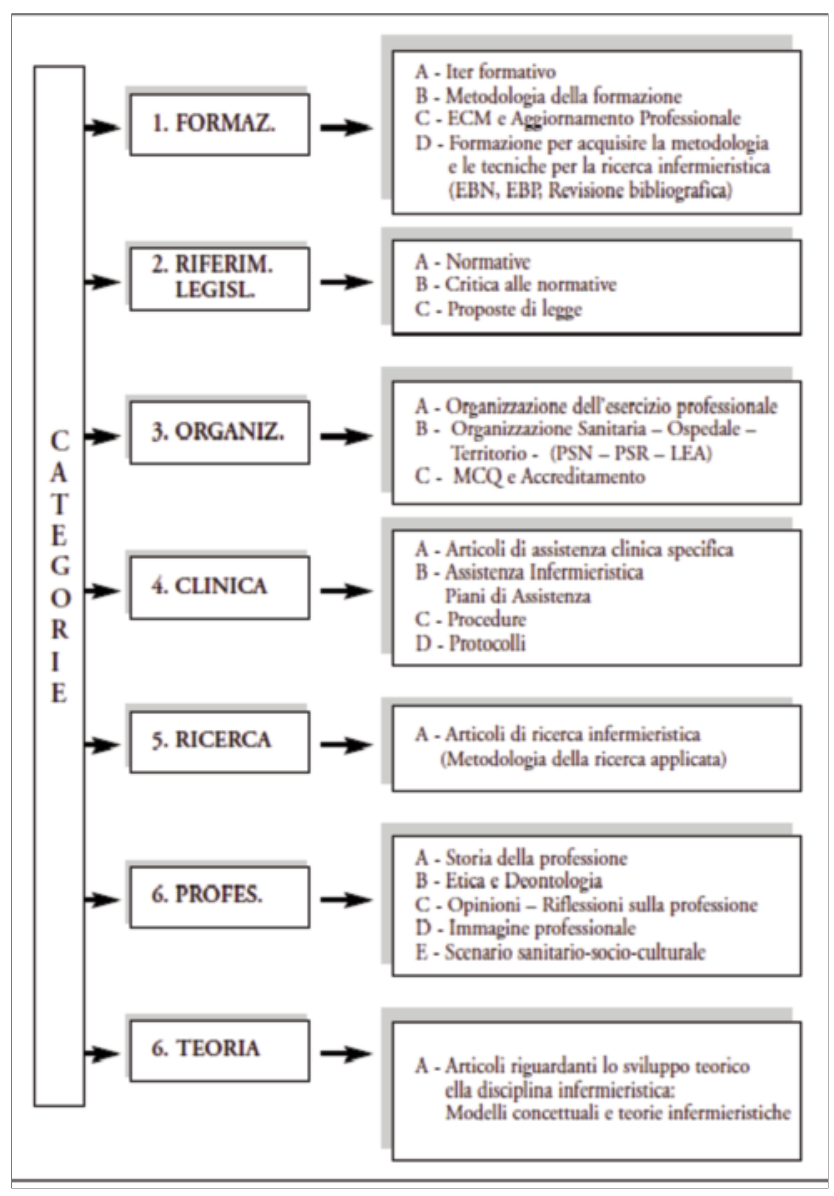

Fig. 1. Codifica delle diverse tipologie di lavori suddivisi in categorie e sottocategorie

\begin{tabular}{|c|c|c|}
\hline Anno & Frequenza & $\%$ \\
\hline 2003 & 218 & 24.6 \\
\hline 2004 & 250 & 28.2 \\
\hline 2005 & 201 & 22.7 \\
\hline 2006 & 218 & 24.6 \\
\hline TOTALE & 887 & 100 \\
\hline
\end{tabular}

Tab. 1. Frequenza articoli per anno

\begin{tabular}{|c|c|c|c|c|c|}
\hline Rivista & Anno & Articoli & \% Anno & $\begin{array}{c}\text { Totale } \\
\text { Articoli }\end{array}$ & \% Rivista \\
\hline \multirow{2}{*}{$\begin{array}{c}\text { Professioni } \\
\text { Ifermieristiche } \\
\text { (trimestrale) }\end{array}$} & 2007 & 25 & 20.49 & & \\
\cline { 2 - 4 } & 2009 & 22 & 18.3 & 74 & 69.65 \\
\hline \multirow{2}{*}{$\begin{array}{c}\text { AlR } \\
\text { (trimestrale) }\end{array}$} & 2007 & 16 & 13.11 & & \\
\cline { 2 - 4 } & 2008 & 17 & 13.93 & \multirow{2}{*}{48} & \multirow{2}{*}{$39: 45$} \\
\cline { 2 - 4 } & 2009 & 15 & 12.29 & & \\
\hline
\end{tabular}

Tab. 2. Distribuzione articoli per rivista ed anno

Per meglio comprendere la modalità di classificazione viene riportata di seguito la legenda dei codici numerici utilizzata per l'analisi dei dati (Fig 2): 


\begin{tabular}{|c|c|c|c|c|c|c|c|c|c|}
\hline Rivista & Anno & Numero & Titolo & Categoria & Sottocategoria & Ricerca & Qualifica & Autore 1 & Autore 2 \\
\hline 1 & 2007 & 4 & $\begin{array}{c}\text { La Diabetologia pediatrica e l'infermiere } \\
\text { case manager }\end{array}$ & 4 & 44 & 2 & 1 & Sabbion P & Renzi E \\
\hline 1 & 2008 & 1 & $\begin{array}{l}\text { I giovani e la professione infermieristica : } \\
\text { indagine... }\end{array}$ & 6 & 65 & 1 & 1 & Giontella M & \\
\hline 2 & 2008 & 2 & Indagine sulla sordità infantile ... & 5 & 51 & 1 & 1 & Corradin L & Fanton $\mathrm{E}$ \\
\hline 2 & 2009 & 2 & $\begin{array}{l}\text { Terapia intensiva aperta : un opzione per- } \\
\text { corribile? }\end{array}$ & 6 & 65 & 1 & 1 & Azzi $R$ & Bambi S \\
\hline
\end{tabular}

Tab.3 Esempio di foglio di calcolo per l'analisi dei dati.

\author{
RIVISTA \\ 1 Professioni Infermieristiche (trimestrale) \\ 2 L'Infermiere (mensile) \\ 3 AIR (Assistenza Infermieristica e Ricerca) (trimestrale) \\ 4 Nursing Oggi (trimestrale) \\ 5 Scenario (trimestrale) \\ CATEGORIE \\ 1 Formazione \\ 2 Riferimenti legislativi \\ 3 Organizzazione \\ 4 Clinica \\ 5 Ricerca \\ 6 Professione \\ 7 Teoria

\section{SOTTOCATEGORIE} \\ 11 Iter formativo \\ 12 Metodologia della formazione \\ $13 \mathrm{ECM}$ e aggiornamento professionale
}

Fig. 2. Legenda dei codici

\section{RISULTATI}

Per il periodo tra il 2003 e il 2006 sono stati analizzati - come già detto in precedenza - 887 articoli distribuiti sulle diverse testate prese in considerazione (Tabella 4). Di questi, 180 non sono scritti da infermieri.

Sul totale degli articoli, escludendo quelli elaborati da autori di altre professioni, abbiamo una distribuzione per categoria come nello schema di seguito (Tabella 5).

Il Grafico 1 invece, evidenzia l'andamento delle pubblicazioni per il periodo esaminato.

Per gli anni tra il 2007 e il 2009 sono stati analizzati complessivamente 24 fascicoli appartenenti alle uniche due riviste infermieristiche censite nei repertori di settore nel periodo considerato. Nel Grafico 2 viene rappresentata la loro distribuzione per cate-

\begin{tabular}{|c|c|c|}
\hline & Frequenza & Percentuale \\
\hline $\begin{array}{l}\text { Professioni Infermieristiche } \\
\text { (trimestrale) }\end{array}$ & 107 & 12.1 \\
\hline Infermiere (mensile) & 366 & 41.3 \\
\hline AIR (trimestrale) & 164 & 18.5 \\
\hline Nursing Oggi & 136 & 15.3 \\
\hline Scenario (trimestrale) & 114 & 12.9 \\
\hline Totale & 887 & 100 \\
\hline
\end{tabular}

Tabella 4 Distribuzione articoli per testata
14 Formazione per acquisire la metodologia e le tecniche per la ricerca 15 Infermieristica 21 Normative

22 Critica alle normative

23 Proposte di Legge

31 Organizzazione dell'esercizio professionale

32 Organizzazione Sanitaria - Ospedale - Territorio (PSN-PSR-LEA)

33 MCQ e accreditamento

41 Assistenza clinica specifica

42 Assistenza Infermieristica - Piani di assistenza

43 Procedure e Protocolli

44 Interventi educativi e promozione della salute

51 Articoli di ricerca Infermieristica applicata

61 Storia della Professione

62 Etica e deontologia

63 Opinioni - riflessioni sulla professione

64 Immagine professionale

65 Scenario sanitario e socio culturale

71 Sviluppo teorico dell'infermieristica: modelli concettuali e teorie

goria.Se si pone maggior attenzione, è possibile rilevare alcune differenze tra le due testate considerate. Infatti per Professioni Infermieristiche compaiono un maggior numero di articoli relativi alla Formazione ed Organizzazione rispetto ad AIR che predilige articoli di Clinica e Ricerca. Va però evidenziato che AIR non ha pubblicato alcun articolo di Teoria nei tre anni valutati.

\section{DISCUSSIONE}

Una prima affermazione che può essere tratta dal presente studio è che si è assistito nel corso degli anni ad un incremento della produzione scientifica da parte degli infermieri da 86 articoli /anno nel 1998 a 222/anno nel 2006 con percentuale di Autori appartenenti alla categoria pari al $78 \%$. Anche se in questo

\begin{tabular}{|c|c|c|}
\hline Categorie & Frequenza & Percentuale \\
\hline Formazione & 95 & 13.4 \\
\hline Riferimenti legislativi & 66 & 9.3 \\
\hline Organizzazione & 117 & 16.5 \\
\hline Clinica & 232 & 32.8 \\
\hline Ricerca & 22 & 3.1 \\
\hline Professione & 164 & 23.2 \\
\hline Teoria & 11 & 1.6 \\
\hline Totale & 707 & 100 \\
\hline
\end{tabular}

Tabella 5 Distribuzione articoli per categoria. 


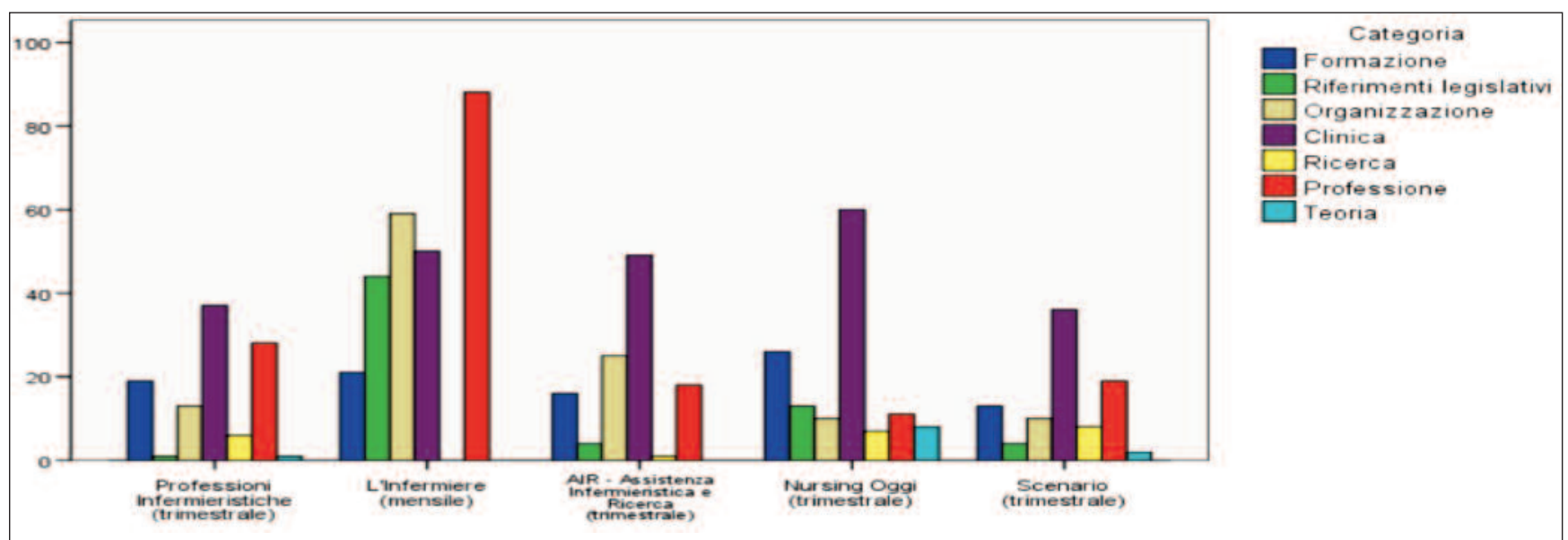

Grafico 1. Andamento pubblicazioni periodo 2003-2006

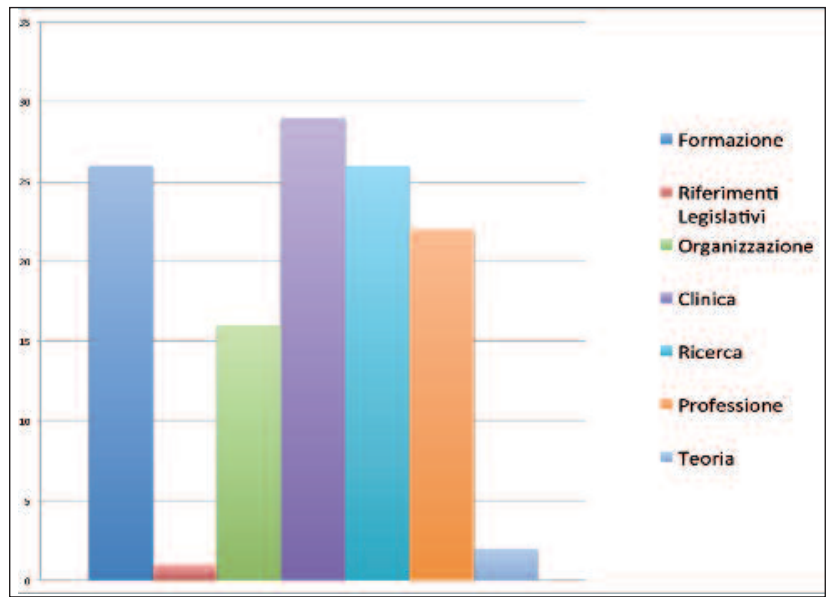

Grafico 2. Distribuzione articoli per categoria 2007-2009.

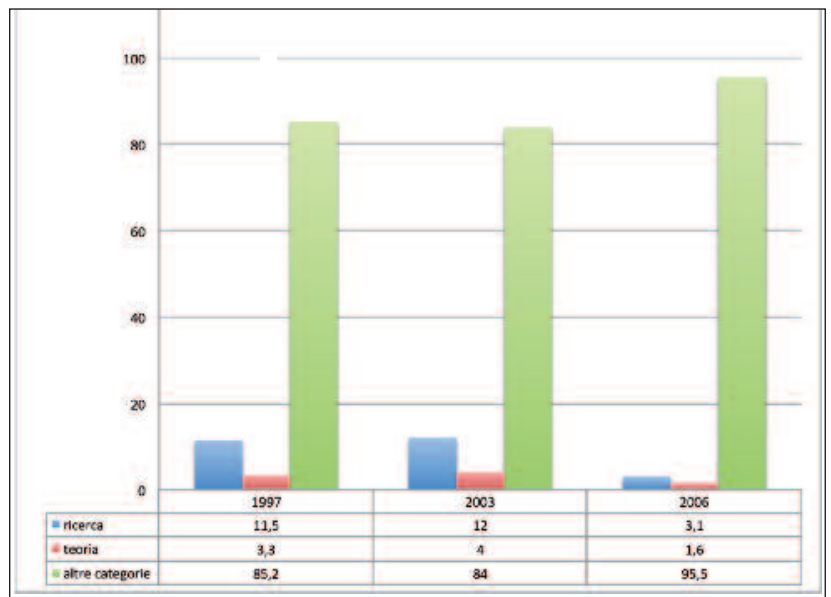

Grafico 3. Andamento articoli teoria e ricerca.

dato non viene considerato il numero di pagine dei singoli articoli, pur tuttavia il divario è evidente.

Infatti all'osservazione della sola proporzione numerica fra gli articoli pubblicati nelle diverse parti di questo lavoro emerge che in circa 30 anni la produzione di pubblicazioni infermieristiche è aumentata di quasi tre volte.

Paragonando i risultati ottenuti in questo lavoro con la pubblicazione del 2005 (Marucci et al.) si può osservare come le categorie già allora fortemente rappresentative si siano ulteriormente rafforzate nel periodo 2003-2006, con la differenza di avvicendamento tra l'area della Clinica verso l'area Professione. Si osserva inoltre la differenza di rappresentatività percentuale delle categorie Ricerca e Teoria tra la presente analisi e l'indagine precedente (Grafico 3).

Questo conferma ulteriormente l'ipotesi che ,nonostante l'incremento della produzione scientifica italiana, globale, gli infermieri scrivono poco di Ricerca e Teoria. E' opinione di chi scrive che comunque siano necessarie alcune considerazioni relativamente ai dati emersi:

- Le linee editoriali delle riviste influenzano inevitabilmente la tipologia delle pubblicazioni

- Può sussistere disomogeneità di classificazione degli articoli

- Nei precedenti lavori non è stata evidenziata la professione degli Autori

La prima considerazione deriva dal fatto che fra le riviste considerate, ad esempio, vi è la testata Scenario organo di stampa dell'ANIARTI (Associazione Nazionale Area Critica e Terapia Intensiva) che pubblica quasi esclusivamente articoli clinici di area specifica.

Il secondo punto è determinato da un limite strutturale del presente studio rispetto ai precedenti in quanto la catalogazione degli articoli è stata effettuata da un diverso gruppo di lavoro con preparazione e culture differenti anche se condotto con l'impegno di individuare le categorie e classificare gli articoli con le medesime modalità. Nel terzo caso, invece l'individuazione dell'autore del lavoro, come ruolo professionale, avrebbe potuto produrre differenti rappresentatività percentuali, avvicinandole o allontanandole ai risultati di questo lavoro.

Questo dato deriva, e sicuramente esorta ad una riflessione, dal fatto che nell'ultimo periodo ci sia stato un incremento delle pubblicazioni in ambito assistenziale con un interesse particolare ad "interventi educativi e promozione della salute" ; chi scrive azzarda il parere che lentamente stia cambiando il 
modello culturale di riferimento. Infatti qualche anno fa si parlava meno di prevenzione. D'altra parte per la figura infermieristica promuovere la salute ai vari livelli è un dovere oltre che morale anche di natura deontologica che permette allo stesso tempo di migliorare lo stato di salute dell'utenza e di ottimizzare le sempre minori risorse a disposizione delle strutture sanitarie. Il tema della formazione rappresenta circa il $21 \%$ del totale degli articoli presi in esame. In questo caso si può dichiarare che negli ultimi 30 anni c'è stato un andamento di tipo altalenante $24 \%$ tra il 1978 e il $1998,12 \%$ tra il 1998 e il 2003 , circa il $14 \%$ dal 2003 al 2006 per arrivare all'attuale $21 \%$. Sicuramente l'assenza di linearità in questi dati è dovuta al fatto che negli anni novanta, con l'introduzione della formazione infermieristica di base in Università, sono stati molti i cambiamenti educativi e curricolari.

Nonostante ciò molti articoli approfondiscono prevalentemente la parte legata al tutoraggio e all'attività tecnica piuttosto che al pensiero scientifico curricolare e allo sviluppo del nursing come scienza per la formazione di una figura professionale competente. Gli argomenti riportati negli articoli oggi riguardano maggiormente bisogni formativi, qualità dei corsi con un attenzione particolare alle attività tecniche di tirocinio.

Per l'area della Ricerca si è apparentemente per questi anni - di fronte ad una situazione altalenante delle pubblicazioni (Professioni Infermieristiche e AIR) relative a questa tematiche (Grafico 4).

Crediamo di poter affermare che nell'ultimo periodo ci sia una tendenza a scrivere di più nella categoria Ricerca, ma i risultati vanno attentamente ponderati in quanto nel presente lavoro sono stati inseriti in questa area oltre agli studi specifici che seguono un metodo di tipo quantitativo, anche studi di validazione di scale e questionari, studi sulla qualità della vita e sulle percezioni dei pazienti relativamente alla sfera socio-emozionale. La scelta di criteri e parametri più ampi ha determinato un aumento quasi del $90 \%$ degli articoli in questa tematica.

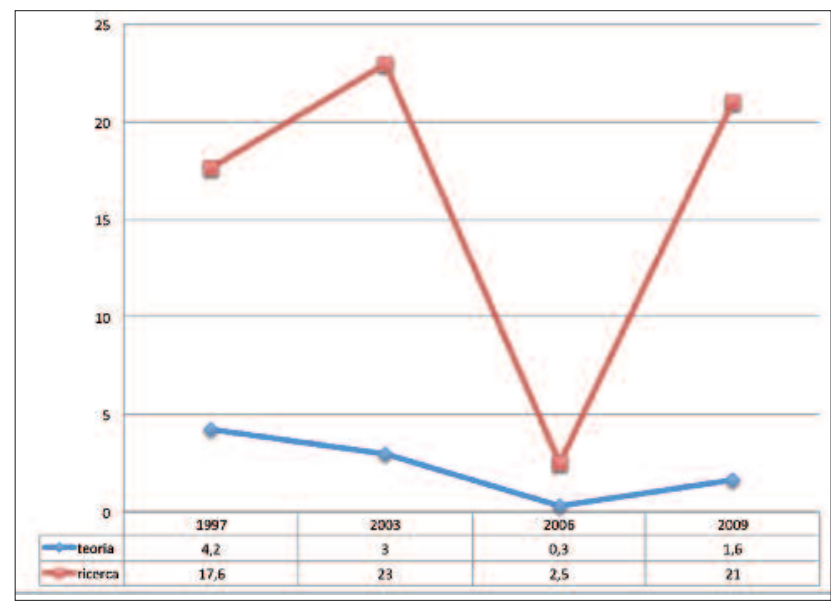

Grafico 4: Andamento nelle testate censite delle categorie Ricerca e Teoria dal 1997 al 2009
Interpretiamo il dato della presenza di molti articoli relativi a validazione di questionari o di scale, come l'evidenza di un interesse da parte degli Autori (e della professione) di trovare, di provare, di fare propri strumenti utili per condurre ricerca prodotti da Colleghi stranieri e studiare fenomeni nuovi dal punto di vista infermieristico, per il nostro Paese. Anche l'aumento di ricerca riguardante la qualità della vita, l'interesse per la percezione dei pazienti relativamente alla sfera socio- emozionale, argomenti poco trattati nell'attuale curriculum del Corso Laurea di Infermiere, può indicare un avanzamento verso un modello infermieristico dettato dalla letteratura straniera ma ancora poco presente nella realtà lavorativa italiana. Analogamente ai lavori precedenti già citati, per essere catalogato nell'area della Ricerca, l'articolo doveva rispondere alle fasi principali del percorso di ricerca [IntroduzioneRevisione della letteratura, Materiali e Metodi, Risultati e Discussione (IMReD)]. Pur comprendendo tutte queste fasi, una ridotta percentuale di articoli era carente nel riportare la revisione della letteratura in quanto povera di riferimenti. Questi articoli sono stati comunque presi in considerazione in quanto nella fase progettuale dello studio non erano stati definiti specifici criteri sulla modalità di presentazione della sezione letteratura salvo la richiesta di una generica presenza della stessa.

Una certa omogeneità nel tempo si è invece riscontrata per la categoria Organizzazione, quasi a sottolineare la costante necessità da parte degli infermieri di dover "scrivere" e quindi "pubblicare" per dimostrare e mettere in luce l'importanza della funzione di management per le strutture sanitarie che l'infermiere ricopre. La Legislazione appare come la categoria meno rappresentata con una significativa riduzione delle pubblicazioni in questo ambito rispetto a studi precedenti. Erroneamente si potrebbe pensare che gli infermieri non scrivano articoli sulle normative, ma è opinione degli Autori che il dato sia riconducibile all'esistenza nello stesso ambito editoriale, a riviste dedicate unicamente ad aggiornamenti di legislazione professionale.

In ultimo non per importanza, troviamo la Teoria che in coerenza con il passato appare scarsamente rappresentata. Si evidenzia che in tutti gli anni esaminati dal 1978 al 2009 questa categoria non è mai andata oltre il $4 \%$. Con scarsa presenza nelle pubblicazioni di un' area o indicazione di specifico modello concettuale di riferimento per lo studio. Anche questo dato ci porta ad una riflessione e richiama quanto discusso da Meleis (2011) sul percorso seguito da una professione nel proprio sviluppo disciplinare e professionale. La stessa Autrice, infatti, riporta che una professione evolve e progredisce attraverso diversi stadi conseguenti: come prima cosa riflette e studia la propria tecnica, poi studia la propria formazione e l' organizzazione, in seguito 


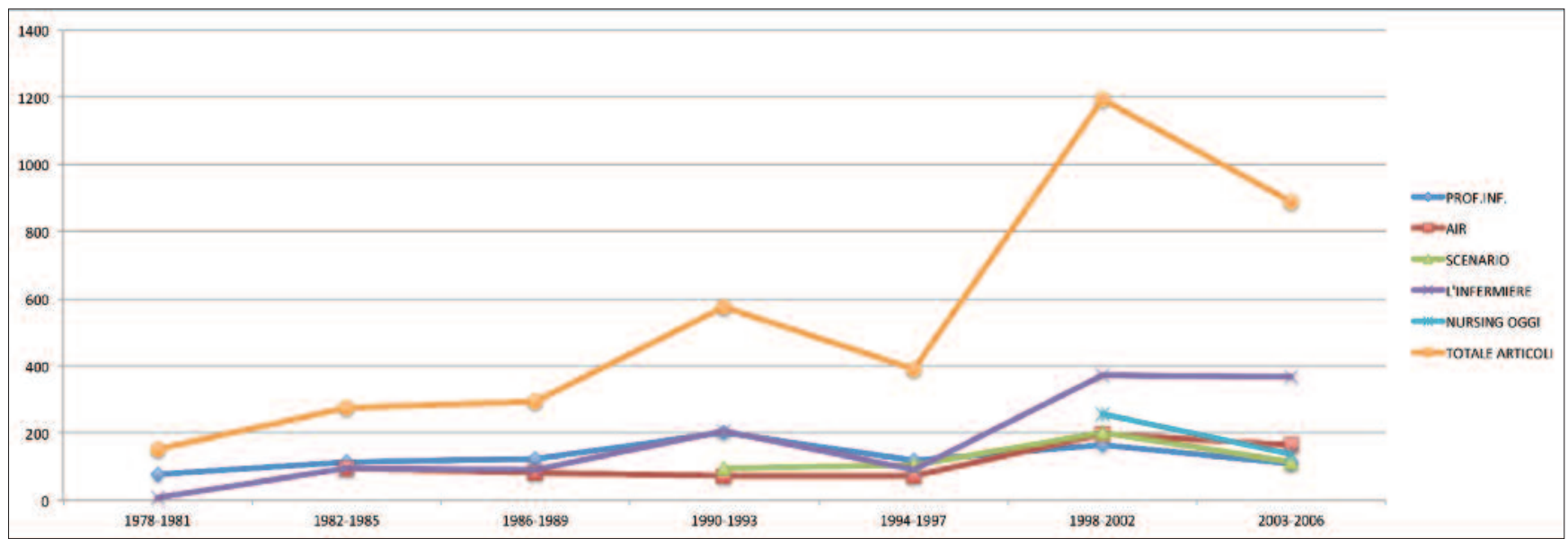

Grafico 5: Andamento quadriennale delle pubblicazioni dal 1978 al 2006.

considera la propria pratica, attività e professionalità per poi giungere alla ricerca ed allo sviluppo di una propria filosofia, alla concettualizzazione per elaborare propri modelli e teorie.

Una professione che non concettualizza e non teorizza la propria specificità condividendola con altri saperi disciplinari, che non sviluppa un sapere proprio deve utilizzare i saperi provenienti da altre discipline con il rischio di rimanere ancorata ad essi ed al tecnicismo piuttosto che all'espansione scientifica e creativa che caratterizzano i saperi e le discipline proprie. Per questo è fondamentale che i risultati a cui si arriva vengano pubblicati dal momento che attraverso le pubblicazioni è possibile dimostrare ed enunciare le proprie conoscenze ed il proprio sapere al fine di poter sostenere che l'infermieristica non è soltanto una scienza applicata (Sansoni, 2005).

Certo è che se ci si soffermasse esclusivamente ad un osservazione in linea teorica, la produzione di pubblicazioni dovrebbe essere costante e con andamento lineare. Un andamento di questo tipo, significherebbe anche la presenza di un gruppo scientifico disciplinare costante nel tempo. Ma i nostri dati lo rappresentano solo in parte. Infatti è possibile notare una diminuzione del divario del numero di pubblicazioni nel tempo con un aumento in termini numerici degli articoli.

Sarebbe necessario un'ulteriore approfondimento per rilevare come nella nostra realtà i cambiamenti professionali influenzino il tipo di pubblicazioni. Non è un caso che nei primi anni del 2000 ci siano numerose pubblicazioni relative alla normativa ed alla formazione, probabilmente i diversi Autori sono stati sollecitati dall'emanazione dei regolamenti attuativi - usciti in quel periodo- relativi alla fomazione specialistica della professione. Si nota inoltre anche un maggior interesse- per articoli relativi all'assistenza clinica specifica, probabilmente a rispecchiare il periodo attuale nel quale si parla sempre piu dell'applicazione delle migliori "evidenze" nella pratica assistenziale.
Il Grafico 5 rappresenta tutta la produzione presa in considerazione dal 1978 al 2006, con raggruppamenti omogenei di quattro anni, troviamo particolarmente significativo anche il costante aumento della produzione infermieristica italiana soprattutto nel periodo 1998- 2002. Crediamo infatti che l'avvento in quegli anni dei primi concorsi universitari per ruoli accademici per la professione infermieristica, abbia avviato la costante necessità di produzione scientifica per scopi diversi ed abbia inciso significativamente sul dato.

Allo stesso modo può far sorgere qualche ipotesi, che necessità di ulteriore approfondimento, la costante diminuzione della produzione di letteratura italiana nei successivi quattro anni.

\section{CONCLUSIONI}

L'impulso che in questi anni ha pervaso la professione dell'infermiere verso una maggiore consapevolezza di necessità di basi scientifiche per lo sviluppo della disciplina e della professione, ha promosso una riflessione sulla mera tecnica ed ha prodotto un significativo interesse per la consultazione dei periodici e delle banche dati assieme alla necessità di scrivere nel proprio ambito.

Sarebbe interessante approfondire maggiormente cosa scriviamo infatti, ci saremmo aspettati una maggiore produzione letteraria riguardo temi rilevanti legati ai diversi metodi di ricerca e come questi possano essere applicati o sono applicati nella realtà assistenziale italiana.

In realtà quanto emerso rispecchia un problema locale della non cultura ad avere fondi dedicati e la naturale insufficienza di investimenti nell'ambito della ricerca, con conseguente mancanza di lavori ma anche con la mancanza di un numero sufficiente di figure del settore, di livello culturale e professionale, preparate per poter educare gli studenti infermieri alla discus- 
sione dei modelli concettuali propri, alla elaborazione mentale di visione teorica e filosofica, alla revisione sistematica della letteratura fin dal periodo formativo di base. In Italia si scrive ancora molto di tecniche, di piani assistenziali e di problematiche inerenti la quotidianità del lavoro. L'immagine che emerge, è in coerenza con quanto già detto in precedenza sullo sviluppo della scala di ricerca come specchio dello sviluppo professionale.

Nonostante siano passati alcuni anni ci si trova a ribadire quanto già dichiarato nel 2005 (Sansoni et al., 2005) ovvero che il grado di affermazione di una professione e di una disciplina sia direttamente collegato alla esistenza di una letteratura più o meno cospicua e che, pertanto, la pubblicazione costituisca uno dei più importanti strumenti di trasmissione della conoscenza e di elevazione della professione. La ricerca con i suoi risultati, non può essere un privilegio per pochi ma uno strumento accessibile a tutti. Sicuramente ancora oggi parlando di ricerca la nostra professione paga la mancanza di una cultura consolidata e di una unione professionale, paga il domino di altre professioni nella formazione e nella direzione assistenziale. Anche se vengono pubblicati articoli di ricerca, spesso questi non rispondono a quella rigorosità metodologica che determina la scientificità del sapere disciplinare. Siamo ancora lontani dal concettualizzare il nostro sapere ma sicuramente consapevoli che l'unico modo per raggiungere questo traguardo è la riflessione sui cardini metaparadigmatici per poter fare ricerca con un chiaro modello concettuale della professione e sviluppare con rigore anche le pubblicazioni su Riviste del settore riconosciute scientificamente.

Dalla produzione sulle Riviste si può vedere lo sviluppo di una professione e di una disciplina. Speriamo venga compreso da tutti noi, Infermieri italiani. Ci auguriamo che nasca anche la consapevolezza che in questo momento storico, la lingua inglese è la lingua della scienza pertanto tutti gli infermieri dovrebbero poterla utilizzare. Vorremmo si comprendesse fino in fondo il valore delle proprie Riviste, delle Riviste come strumento di diffusione del sapere, strumento di discussione, di condivisione scientifica, strumento di visibilità del beneficio dell'assistenza e dell'impegno di una professione.

Si osserva inoltre il cambiamento di priorità di alcuni Autori italiani che al fine di avere maggiore visibilità (H Index) e presenza di articoli su Riviste ad elevato Impact Factor, scelgono di pubblicare su Riviste straniere appartenenti anche ad altri settori dimenticando la necessità di scambio interno quale priorità per far crescere la professione infermieristica del Paese.
Diversamente sarebbe necessario chiedere con fermezza, ed accettare senza alcun complesso di inferiorità o paura di esporsi, indicatori bibliometrici propri, che possano dare prestigio e rafforzare la visibilità del sapere del settore nella piena consapevolezza del bisogno di un continuo sviluppo nel Paese e internazionalmente

Abbiamo necessità di Agenti di cambiamento.

\section{BIBLIOGRAFIA}

Burrows, DE., McLesh, K. (1995). A model of research -based prctice. Journal of clinical Nursing 4(3) 243-247.

Cantarelli, M. (2002). Ricerca Infermieristica sfida di ieri, necessità di oggi. In italia come si è sviluppata la ricerca infermieristica ?. Atti Convegno CNAI Roma 18-20 Ottobre 2001. 17-34

French., P. (1999). The devolpment of evidence -based nursing. Journal of Advanced Nursing. 29(1) 72-78.

Hunt, JM. Guest Editorial.( 1996). Journal of Advanced Nursing. 23 (9) 423-425

Kitson, A., Ahmed, LB., Harvey, G., Seers, K., Thompson DR. (1996). From research to practice: one organisational modelfor promoting research - based practice. Journal of Advanced Nursing. 23 (9) 430-440

Marucci, AR., Sansoni, J., Pierantognetti, P., Luzzetti, E. (2005). La produzione scientifica italiana attraverso una analisi di cosa pubblicano gli infermieri. Seconda parte: anni 1998-2003. Professioni Infermieristiche, 58 (4), 236-245

Meleis, A.I. (2011). Theoretical nursing: Development and progress (5th Ed.). Philadelphia, PA: Lippincott Williams \& Wilkins.

McCarthy, G., Hegarty, J., O Sullivan, D. (2006) Nursing research in Irlend. Annual review of nursing research. 295- 331.

Polit, DF., Beck, CT. (2009) International difference in nursing research 2005-2006. Journal of Nursing Scolarship 41(1) 44-53

Sansoni, J., Marucci, AR., Spalluto, M., Pierantognetti, P., Giustini, M. (2005). Una fotografia delle aree di produzione scientifica infermieristica italiana attraverso una analisi di cosa pubblicano gli infermieri. Prima parte: anni 1978-1997. Professioni Infermieristiche 58(4), 229-235.

Sansoni, J., Comerci, S., Marucci, AR. (2006) Infermieri e ricerca: analisi di due riviste infermieristiche internazionali. Professioni Infermieristiche 59(1), 4148.

Silva, LM., Oliveira, NR., Frota, MA., Fialho, AV. (2008). International research in nursing: thematic and methodological tendencies. Revista Brasileira de Enfermagement 61(5) 314-322.

Zanotti, R., Pecile, A. (2002). La ricerca infermieristica in Italia nel periodo 1998-2001. Professioni 